\title{
Beobachtungen über das Verhalten einiger wirbelloser Thiere gegen galvanische und faradische Reizung.
}

\author{
Von
}

Dr. Wilibald Nagel

in Tübingen.

Bei Wasserschnecken und Copepoden beobachtete ich ein auffallendes Verhalten gegenüber elektrischen Strömen, welches den durch Versuche an Wirbelthieren abgeleiteten Gesetzen elektrischer Erregung auf den ersten Blick zu widersprechen scheint. Ob diese Abweichung nur eine scheinbare, durch die ungleichen Versuchsbedingungen herbeigefübrte ist, oder ob man hier wirklich annehmen muss, dass die bei diesen Thieren giltigen Gesetze audere sind, als die der menschlichen Nervenphysiologie, lasse ich vorläufig dahingestellt, und führe einfach die Thatsachen an.

\section{Versuche an Limnaeus und Planorbis.}

Wenn man in der Nähe einer in flachem Wasser kriechenden Wasserschnecke die zwei Poldrähte einer constanten Stromquelle ins Wasser taucht, so bemerkt man, dass die beiden Elektroden ungleich stark anf das Thier einwirken, und dass die eine derselben bei Stromschluss, die andere bei Stromöffnung wirksam ist. Die Wirkung besteht in lokaler Contraktion des Schneckenkörpers.

Die verwendeten Ströme waren schwach. Ein kleines Grenetsches Tauchelement (wirksame Kohlenoberfläche ungefähr $60 \mathrm{~cm}^{2}$ ) mit einer durch mehrfachen Gebrauch geschwächten Chromsäurefüllung wurde mit den 2 Elektroden verbunden, welche gewöhnlich aus diinnem Platindraht bestanden. Diese wurden im Mittel nicht über $1 \mathrm{~cm}$ tief eingetaucht. Der schon an sich schwache Strom wurde noch dadurch bedeutend abgeschwächt, dass die Elektroden $5-10 \mathrm{~cm}$ von einander eingetaucht wurden. Sollte die Reaktion 
Ueber das Verhalten einiger wirbelloser Thiere geg. galvanische etc. Reizung. 625

stärker ausfallen, so verwendete ich zur indifferenten Elektrode ein Stiick Platinblech $\left(2 \times 3 \mathrm{~cm}^{2}\right)$.

\section{Versuch I.}

Kathode dem Kopf der Schnecke nahe eingetaucht; Anode nachträglich in Entfernung (ca. $10 \mathrm{~cm}$ ) eingetaucht. Keine $Z u c k u n g$ oder sonstige Reaction.

\section{Versuch II.}

Dieselbe Anordnung, aber beide Drähte eingetaucht. Jetzt wird die entfernte Anode aus dem Wasser gezogen. Die dem Kathodendraht zunächst liegenden Theile der Schnecke zucken momentan vor diesem zurück. (Kathoden-Oeffnungs-Zuckung.)

\section{Versueb III.}

Dieselbe Anordnung, aber die Kathode wird herausgezogen. Der Erfolg ist wie bei II. Dasselbe wird erreicht, wenn beide Elektroden im Wasser bleiben, und der Strom an anderer Stelle unterbrochen wird.

\section{Versuch IV.}

Anode dem Thiere nahe, Kathode nachträglich in Entfernung eingetaucht. Dabei heftiges Zurückzucken vor der Anode, dauernde abstossende Wirkung der Anode. Anoden-Schliessungsuckung stärker als Kathoden-Oeffnungszuckung.

\section{Versuch V.}

Dieselbe Anordnung, beide Drähte eingetaucht; nachträglich Anode oder Kathode herausgezogen, oder an anderer Stelle der Strom unterbrochen. Keine Zuckung, Aufhören des Anodenreizes.

Will man diese Resultate in einer Formel, ähnlich der sog. $\mathrm{Br}$ e n n er'schen Normalformel ausdrücken, so würde dieselbe lauten:

\begin{tabular}{l|c|c|c|c}
\hline Stromintensität & Anod.-Schl. & Anod.-Oeffn. & Kath.-Schl. & Kath.-Oeffn. \\
\hline schwach & $\mathrm{Z}$ (uckung) & $\mathrm{R}(\mathrm{ahe})$ & $\mathrm{R}$ & $\mathrm{R}$ \\
\hline stark & $\mathrm{Z}$ & $\mathrm{R}$ & $\mathrm{R}$ & $\mathrm{Z}$
\end{tabular}

Das Gesetz für die Zuckung vom Nerven aus beim Frosche lautet: 


\begin{tabular}{c|c|c|c|c}
\hline Stromintensität & Anod.-Schl. & Anod.-Oeffn. & Kath.-Schl. & Kath.-Oeffn. \\
\hline schwach & $\mathrm{R}$ & $\mathrm{R}$ & $\mathrm{Z}$ & $\mathrm{R}$ \\
\hline stark & $\mathrm{R}$ & $\mathrm{Z}$ & $\mathrm{Z}$ & $\mathrm{R}$ \\
\hline
\end{tabular}

wobei immer die erste der beiden Stromintensitäten so stark gedacht ist, dass sie im ersteren Falle (Limnaeus) bei Oeffunng an der Kathode, im zweiten Falle (Frosch) bei Oeffnung an der Anode nicht ausreicht, um Zuckung zu bewirken.

Als empirischer Satz formulirt: Bei Stromsehlus serfolgt Reizung nurander Anode, bei Stromöfnung nurander Kathode. DieAnodenreizungist die stärkere.

Der entsprechende Theil des von Pflüger für Wirbelth i er e am frei präparirten Nerven festgestellten Gesetzes würde lauten: Bei Stromschluss erfolgt Reizung nur an der Kathode. Bei Stromöffnug nur an der Anode. Die Kathodenreizung istdiestärkere.

Die Körperstellen, an welchen die elektrische Reizung wirksam ist, fallen zusammen mit denjenigen, welche auch auf schwächere $\mathrm{ch}$ e $\mathrm{mis} \mathrm{ch}$ e Reize reagiren, welche also Schmeckwerkzenge enthalten. Säuren, Alkalien und dergl. dürfen zu Versuchen uiber die Lokalisation des chemischen Sinnes nicht, oder nur in hochgradiger Verdïnnung verwendet werden. Verwendet man aber z. B. Chininbisulfat, Strychninnitrat, Saccharin, schwache Lösungen von Kaliumbichromat, so findet man eine ganz bestimmte Gegend des Körpers durch diese Stoffe reizbar; dieselben sind es, welche sich vor der differenten Elektrode energisch zuriickziehen. Am empfindlichsten sind die Lippen oder oberen Mundlappen, dann folgen mit etwas geringerer Empfindlichkeit die Fühler und der vordere Rand des Fusses (Unterlippe), sodann der ganze uibrige Fussrand und die Vorderseite des Kopfes zwischen den Fühlern. Ein geringes Maass von Empfindlichkeit zeigt noch der Mantelrand, an ganzen übrigen Körper jedoch erfolgt Reaktion auf chemische wie elektrische Reizung erst spät, langsam und unsicher, man ist nie sicher, ob die schliesslich doch eintretende Reaktion nicht Folge der Ausbreitung des Reizes ist. Bei einem 
an der Wasserfläche schwimmenden Limnaeus kann man die Elektrode tief in den Raum zwischen Rücken und Mantel einführen, ohne zu reizen; und andererseits kann man diesen Raum mittelst einer Pipette mit Kalinmbichromatlösung anfüllen, ohne dass das Thier früher als nach $10-20$ Sec. langsam sich zurückzuziehen beginnt. Ein Tröpfehen der Lösung, an den Fussrand oder Fühler gebracht, lässt diese Theile sofort sich energisch zusammenziehen.

\section{Wirkung des Induktionsstromes.}

Verbindet man die Enden der sekundären Spirale eines Induktionsapparates mit Elektroden, bestehend aus dünnem Platindraht, und nähert diese einer Wassersehnecke, so beobachtet man, dass beide Elektroden reizend wirken, die eine aber beträchtlich stärker, als die andere. Durch Verschiebung des Schlittens am Dubois-Reymond'schen Apparate kann man eine Stromstärke finden, bei welcher die Reizwirkung einer Elektrode ganz verschwunden ist, während die andere noch deutlich abstösst. Letztere, die differente Elektrode, ist diejenige, welche auf Jodkaliumstärkekleisterpapier den stärkeren blauen Streifen zeichnet, somit die Elektrodemit vorwiegender Anodenwirkung.

Die Ursache der Ungleichbeit der Wirkung beider Elektroden ist, wie leicht begreiflich, die, dass der primäre Strom mittelst eines gewöhnlichen Wagner'schen Hammers bewirkt wurde. Bei Verwendung dieses Apparates wird der Schliessungsinduktionsstrom durch die verzögernde Wirkung des in der primären Rolle zustande kommenden Extrakurrent-Stromes in der Weise modificirt, dass er langsamer zu seiner vollen Höhe ansteigt, als der Oeffnungsstrom. Daher die geringere physiologische Wirkung.

Anmerkung. Folgendes Schema veranschaulicht die einzelnen Phasen der Induktionswirkung während einer Oscillation des Unterbrechers in ihrer relativen Stärke.

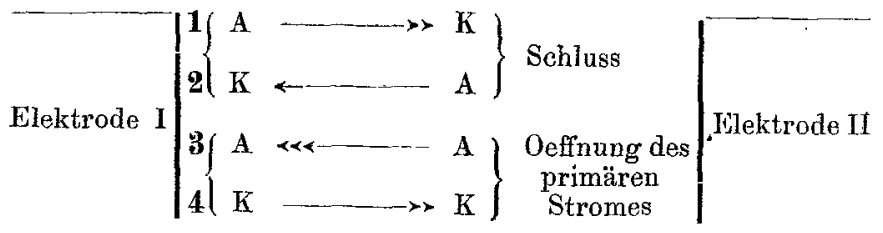

Die Pfeile bedenten die Stromrichtungen, ihre Form deatet augleich die Stärke an. Dass Strom 1 und 3 stärker sind als 2 und 4 (bez.), kommt 
daher, dass das Eintreten des Induktionsstromes das wirksamere an diesem ist, nicht das Vergehen. Dass Strom 3 und 4 stärker sind, als Strom 1 und 2 (bez.), kommt daher, dass 3 und 4 dem an sich stärkeren Oeffnungsinduktionsstrom angehören. Es ist somit erklärlich, dass trotz des steten Wechsels der Stromrichtung doch eine wirksame Stromrichtung vorherrscht, und dass die eine Elektrode mehr Anodenwirkung, die andere Kathodenwirkung hat.

Da beim constanten Strome die Anode bei Stromschluss wirksam ist, muss es beim Induktionsstrom natürlich ebenso sein, was die erwähnte Prüfung mittelst der Jod-Stärke-Reaktion bestätigt.

Es ist leicht zu zeigen, dass die Ungleichbeit der Elektroden durch die Stromunterbrechungsvorrichtung bedingt ist. Man schaltet statt des Wagner'schen Hammers den Helmholtz'schen Unterbrecher ein, welcher an Stelle der Unterbrechung den Eintritt eines kurzen Nebenschlusses setzt. Dadurch kommt anch der Oeffnungsextrastrom zustande und nun haben Oeffnungs- und SchliessungsInduktions-Strom denselben Verlauf und damit dieselbe physiologische Wirksamkeit. Die letztere ist bekanntlich sehr gering; immerhin reagiren die Schnecken auf Ströme, welche auf meiner Zunge keine Empfindung hervorrufen, noch sehr deutlich. Die beiden Elektrodèn verhalten sich jetzt ganz gleich, beide stossen in gleichem Maasse ab.

Limnaeus stagnatis und Planorbis corneus zeigen in ihrem Verhalten gegen constanten Strom einige kleine Unterschiede. Letzterer ist im ganzen etwas weniger reizbar, namentlich auch seine Fühler (dasselbe gilt von chemischen Reizen). Besonders ist auffallend, was bei Limnaeus nur zuweilen angedentet ist, dass die Fíhler des Planorbis auch von der Anode nur kurze Zeit gereizt werden; sehr bald nach Stromschluss nähert sich der Fühler zuweilen zufällig ohne Reizerscheinungen der Anode, während der Lippensaum dieselbe andanernd vermeidet.

Merkwürdig war die Wirkung des constanten Stromes auf die ausgestreckten Fühler des Planorbis in einigen Fällen: Auf einer Seite der Fühlerspitze (einige $\mathrm{mm}$ von ihr entfernt) tauclte ich den Kathodendraht ein, danach auf der anderen Seite den Anodendraht. In diesem Moment schlägt der Fühler wie eine Galvanometernadel nach der Kathodenseite hin aus. Beim Herausziehen der Kathode schlägt der Fühler nach der Anode hin aus. Der Versuch gelingt jedoch nicht immer. 


\section{Versuche an Cyclops.}

Auf die Thatsache, dass Copepoden die eine Elektrode des faradischen Stromes fliehen, die andere aufsuchen, hat mich Herr Dr. J. Vos seler aufmerksam gemacht, welcher dieselbe an Süsswasser- und Meeres-Copepoden beobachtet hat.

Wenn man zwei etwas umfangreiche Elektroden in das Wasser setzt, welches Cyclopsarten enthält, so sammeln sich in wenigen Sekunden die meisten Krebse um die eine der Elektroden. Die andere wird sichtlich gemieden. Die einzelnen Thiere kommen aus entfernten Theilen des Gefässes, aus ibren Verstecken, herbeigeschwommen, um zu der Elektrode zu gelangen, und umschwimmen dieselbe in Bewegungen, welche mehr als gewöhnlich den Charakter von krampfhaften, zuckenden haben.

Die Elektrode, um welche sich die Copepoden sa m meln, ist auffallenderweise diejenige, welche die empfindlichen Theile der Schnecken abstösst, also diejenige mit vorwi ge $\mathrm{n}$ de r A n od e n wirk ung.

Der Moment des Eintritts des faradischen Stromes ist besonders wirksam; momentan zucken die zwischen den Elektroden befindlichen Thiere nach der einen derselben hin, so dass man an ihnen die Richtung des (wirksamen) Stromes. wie an einem Galvanoskop erkennen kann. Die Intensität des Stromes muss iibrigens beträchtlich höher sein als zur faradischen Reizung der Sehnecken. Ich experimentirte daher mit ganz eingeschobenem Schlitten. Eine nachhaltige Schädigung der Thiere durch die Versuche war nicht zu bemerken.

Zum Vergleiche prüfte ich das Verhalten der Larven von B ombina tor igne us gegen galvanischen und faradischen Strom. In beiden Fällen scheinen die Elektroden nicht ungleich zu wirken, es erfolgt nicht Ansammlung um eine der Elektroden. Um überhaupt sichtbar zu wirken, muss der Strom sehr viel stärker sein, als bei den Schnecken. Dann aber tritt auch rasch völlige Lähmung ein.

Ich vermisste ferner die polare faradische Reizung bei 0 stracoden, sowie dem kleinen Wurm $\mathrm{T}$ ubifex rivulorum. In beiden Fällen sind aber die Versuchsbedingungen ungïnstige. Beide Elektroden wirken reizend und werden geflohen, es liess sich aber nicht feststellen, ob in ungleichem Maasse. Ich beabsichtige Fort. 
setzung der Versuche an grösseren Würmern und an Wasserinsekten.

Nach Abschlass vorstehender Versuche fand ich eine bemerkenswerthe Parallele za denselben in der interessanten Abhandlung von M. Verworn, Die polare Erregung der Protistendureh dengalvanisehen Strom, in Pflügers A rehiv für Physiologie Bd. 45, 1889 und Bd. 46, 1890. Verworn beobachtete bei einer grossen Zahl von Protisten polare Erregung des Zellprotoplasmas, welche sich in Contraktion, bei stärkerer Reizung in Zerfall des der erregenden Elektrode zugewendeten Körpertheiles äussert. Folge dieser polaren Erregung ist Einstellung der Körperlängsaxe in die Stromrichtung und Bewegung nach einer der beiden Elektroden hin. Einstellung: mit den Vorderenden nach der Anode wird als positiver Galva notropismus bezeichnet, die umgekehrte Einstellung als nega-, tiver Galvanotropismus. Der letztere scheint der bäufigere zu sein, und ist Folge der Anodenschliessungserregung. Diese ist höchst auffälliger Weise den Protisten mit den genannten Wasserschnecken gemein, bei welchen jedoch die Vorgänge der Erregung und Contraktion viel complicirterer Art sind, und offenbar mit Betheiligung des Nervensystemes sich abspielen. Ob etwa die ganzen Schneckenkörper sich mit negativem Galvanotropismus einstellen, habe ich noch nicht feststellen können; sicher ist nur, dass sie die Nähe der Anode vermeiden.

Die erwähnten Beobachtungen an den Copepoden sind nach der von Verworn benützten Bezeichnungsweise Erscheinungen des positiven Galvanotropimus. Die einfache und gewiss zutreffende Art, wie $\mathrm{V}$ erworn mechanisch die Axeneinstellung der Protisten erklärt, reicht wohl für die Copepoden nicht aus, vielmehr wird hier an reflektorische Einstellung durch Vermittelung des Nervensystemes zu denken sein.

Sehr auffallend ist das Ausbleiben der polaren Erregung bei den Bombinator-Larven, welche ich als Vertreter der Wirbelthiere untersuchte. Der Umstand, dass trotz der ähnlichen Hautbeschaffenheit bei diesen und den Schnecken die Reaktionen so sehr ungleich sind, lassen wohl daran denken, dass bei den Sehnecken wirklich die Umkehrung des Pfl iu g er'schen Gesetzes für elektrische Nervenreizung vorliege, wie nach Verwo rn's Untersuchungen die Reizgesetze bei den Protisten für 2 Gruppen derselben verschiedene 
sind. Zur Entscheidung der Frage müsste man womöglich bei einem Wasserwirbelthier Galvanotropismus nachweisen, der dann voraussichtlich ein positiver wäre. Damit würde man imstande sein, auszuschliessen, dass die Reaktion der Schnecken auf direkter Muskelreizung allein beruhe und würde ferner den zweifelhaften Faktor eliminiren, der dadurch in die Frage hineinkommt, dass man bei Reizung eines ganzen Thieres nicht weiss, ob das wirksame die Erregung centrifugaler oder centripetaler Nerven oder subcutan gelegener Ganglien sei. Gerade bei den Schnecken wäre auch dás letztere ernsthaft in Betracht zu ziehen, weil die als reizbar sich erweisenden Hautstellen üher starken Ganglienanhäufungen sich befinden. 